\title{
Therapeutic Peptides as Emerging Options to Restore Misguided Host Defence and Homeostasis: From Teaching to Concept to Clinic
}

\author{
Oliver Schwardt, Christina Lamers, Clément Bechtler, and Daniel Ricklin*
}

\begin{abstract}
Among the many molecular entities suitable for therapeutic use, peptides have emerged as a particularly attractive option for academic drug discovery and development. Their modular structure and extendibility, the availability of powerful and affordable screening platforms, and the relative ease-of-synthesis render therapeutic peptides highly approachable for teaching and research alike. With a strong focus on the therapeutic modulation of host defence pathways, including the complement and renin-angiotensin systems, the Molecular Pharmacy group at the University of Basel strongly relies on peptides to introduce students to practical aspects of modern drug design, to discover novel therapeutics for immune and inflammatory diseases, and to expand on options for the preclinical development of a promising drug class. Current projects reach from student-driven iterative design of peptidic angiotensin-converting enzyme inhibitors and the use of phage display technology to discover novel immune modulators to the development of protective peptide coatings for biomaterials and transplants and the structure-activity-relationship-guided optimization of therapeutic peptide drug candidates in late-stage clinical trials. Even at the current stage, peptides allow for a perfect circle between pharmaceutical research and education, and the recent spark of clinical applications for peptide-based drugs may only increase the value and relevance of this versatile drug class.
\end{abstract}

Keywords: ACE inhibitors $\cdot$ Complement $\cdot$ Host defence $\cdot$ Phage display $\cdot$ Peptide therapeutics

\section{Coming Full Circle: Peptides as Central Element of Pharmaceutical Research and Education}

Uniquely situated between low-molecular-weight (LMW) drugs and 'biologics' such as antibodies, therapeutic peptides represent a versatile class of pharmaceutical compounds consisting of amino acid chains (usually less than 40 residues).[1] Originally, therapeutic peptides have been gained solely from natural sources such as animal tissue as exemplified by the isolation and first clinical use of insulin in the 1920s in diabetic patients. Progress in sequence elucidation, chemical synthesis and biotechnology allowed for the rational design, de novo synthesis and large-scale production of peptidic drugs, leading to the approval of more than 100 therapeutic peptides spanning various clinical indications. ${ }^{[2]}$ When compared to the development of LMW drugs, which often require synthesis routes tailored to a specific class, the modular structure and defined, automatable production (i.e. solid-phase peptide synthesis; SPPS) render peptides more easily available, particularly for academic institutions. In addition, in vitro selection methods, such as phage and mRNA display, enable the generation of large peptide libraries for screening purposes. ${ }^{[3]}$ Since its first report, ${ }^{[4]}$ phage display has evolved into an accelerator of hit generation for both antibody and peptide drugs..$^{[5]}$ The library is displayed on an outer membrane protein of the phage, such as pIII or pVIII, and can reach a diversity of $>10^{9}$ molecules. By linking the phenotype of a peptide library with its genotype, phage display facilitates deconvolution of identified hits. The advantage of phage display, e.g. compared to DNA-encoded libraries and mRNA display, is the use of bacteriophages as vector, which enables amplification of affinity-selected phages in E. coli. In recent years, the introduction of chemically-modified phage libraries that enable in situ macrocyclization to render peptides more stable and drug-like further increased its usability. ${ }^{[6]}$ In mRNA display, a peptide library is covalently linked to its encoding mRNA during the translational process. The use of in vitro transcription and translation allows for easy incorporation of noncanonical amino acids and avoids transformation steps that can limit the diversity of phage libraries. ${ }^{[7]}$

Their high clinical relevance, accessibility and affordability greatly facilitates an integration of peptide drugs into the practical training of students while, at the same time, enabling academic drug discovery (Fig. 1). At the Department of Pharmaceutical Sciences of the University of Basel, therapeutic peptides have not only evolved to a recurrent theme in teaching and research but also provide valuable anchor points to Basel's vibrant academic and industrial environment.

\section{Therapeutic Peptides in Teaching: The Lab Course 'Modern Drug Design'}

Among the most valuable features of peptide drugs is that initial lead compounds can often be derived from natural sources (e.g. scissile loops as protease inhibitors) and further optimized or developed into LMW drugs. Inhibitors of the angiotensinconverting enzyme (ACE) are prominent examples, which we employ for educational purposes. The laboratory course 'Modern Drug Design' offers bachelor students in pharmaceutical sciences a hands-on experience in the rational design, synthesis, and biological evaluation of potential drug candidates (Fig. 2). ${ }^{[8]} \mathrm{ACE}$, an endopeptidase, is a central component of the renin-angiotensin- 


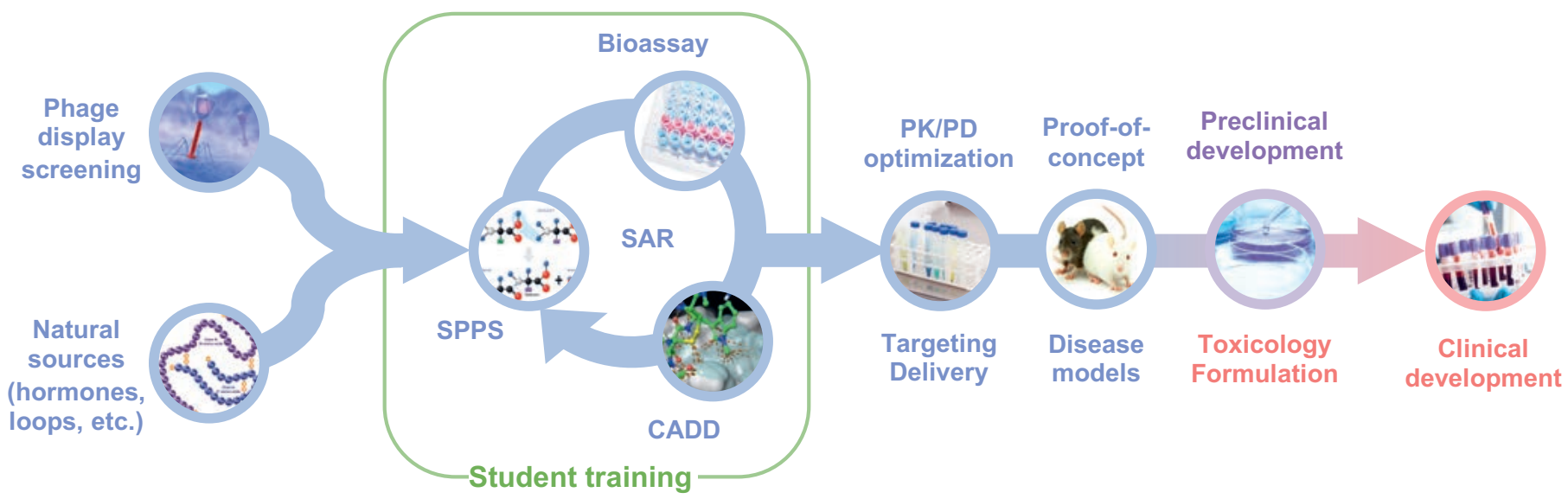

Fig. 1. Opportunities for integrating peptide therapeutics in academic research and education. Phage display libraries or natural proteins often serve as source or inspiration for peptide drug leads, which are then optimized through iterative cycles of solid-phase peptide synthesis (SPPS), evaluation in bioassays and computer-aided drug design (CADD) to obtain structure-activity relationships (SAR). Whereas initial development steps can often be conducted in academic labs, preclinical and clinical development is typically performed by industrial partners.

aldosterone system (RAAS), the body's most important blood pressure-regulating mechanism. ${ }^{[9]}$ Within the RAAS cascade, ACE mediates the conversion of angiotensin I to the vasoconstrictor angiotensin II but also mediates the degradation of bradykinin. Consequently, inhibiting ACE impairs the formation of angiotensin II, thereby lowering blood pressure by reducing smooth vascular muscle contraction and peripheral resistance. ${ }^{9]}$ A nonapeptide inhibitor isolated from a snake venom, called teprotide, served as lead compound for ACE inhibitors but was not orally active. ${ }^{[10]}$ However, systematic structure-activity relationship (SAR) analysis of peptidic ACE inhibitors and synthetic derivatives thereof led to the development of captopril, the first orally active ACE inhibitor approved for antihypertensive therapy. ${ }^{[11]}$ Derivatives with improved efficacy and adverse effect profiles, such as enalapril, lisinopril, and ramipril, have subsequently been introduced to the market.[9b]

Developing next-generation ACE inhibitors is, of course, not the aim of the 'Modern Drug Design' course, but this drug class offers a highly illustrative example for iterative drug development. In the first part of the laboratory course, small peptidic ACE inhibitors (usually tripeptides with $\mathrm{C}$-terminal proline) are identi- fied by computer-aided drug design based on the experimental structure of the ACE-enalapril complex, and their potency is estimated in silico. Alongside good affinity and selectivity, ideal compounds should also show a balanced pharmacokinetic profile (PK; e.g. polar surface area, number of hydrogen-bond donors and acceptors). Thereafter, the most promising ACE inhibitor candidates are synthesized using SPPS. Starting from the preformed Fmoc-Pro-Trityl-solid support, tripeptides are obtained after two cycles of $N$-deprotection and coupling using natural and/or non-natural amino acid building blocks. The peptides are then cleaved from the solid phase and analyzed by HPLC and MS. Finally, the crude peptides are purified by preparative LCMS. In the final stage of the lab training, the ACE inhibitory activity of the peptides is determined in a competitive fluorometric assay based on chromogenic substrate cleavage (i.e., Abz-Gly( $p$-NO $\mathrm{NO}_{2}$-Phe)-Pro). The best inhibitors obtained in the practical course usually show $\mathrm{IC}_{50}$ values in the single-digit micromolar range, which is about 1000 -fold weaker than the potency of commercial ACE inhibitors such as enalapril and captopril (Fig. 2). ${ }^{[12]}$ The communication of the results to the next class of students allows for an iterative improvement of the design strategy.
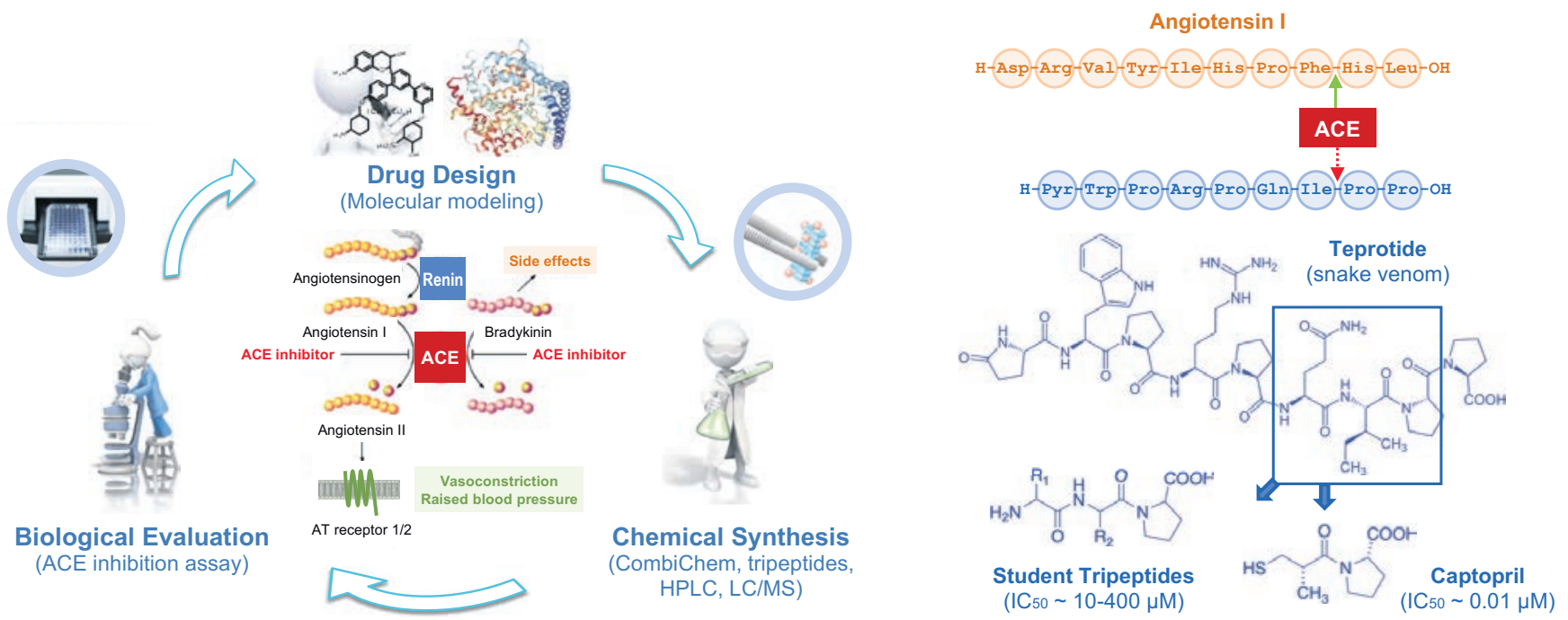

Fig. 2. Peptide-focused practical course 'Modern Drug Design' at the University of Basel. Based on the structure of enalapril-complexed angiotensin-converting enzyme (ACE), students use molecular modeling to design tripeptidic ACE inhibitors, synthesize them in the lab, evaluate their ACEinhibitory potency and report results to subsequent student groups to enable iterative lead optimization. 


\section{Therapeutic Complement System Inhibition Using Peptide Drugs}

In contrast to the educational project above, efficacy, selectivity and PK properties become highly relevant in drug discovery research. Compared to LMW drugs, peptide therapeutics typically feature low oral bioavailability and cell permeability but often show beneficial selectivity and safety profiles. In addition, their size facilitates a use as protein-protein interaction (PPI) inhibitors. These properties render peptide drugs ideally suited as therapeutic modulators of the plasma-based cascade systems that are central to host defence pathways, including the complement, coagulation and contact systems. While providing rapid protection against breached barriers and microbial invasion (e.g. after injury), the limited specificity of these defence pathways may turn them against the host and contribute to clinical complications in age-related, autoimmune or thromboinflammatory conditions. ${ }^{[13]}$ During organ transplant rejection or COVID-19, for example, hyperacute activation of several pathways may induce tissue damage. ${ }^{[14]}$ In such cases, rapid inhibition of the PPI-driven cascade systems becomes critical, whereas bioavailability and permeability are of low importance. With a focus on the complement system, our group investigates therapeutic strategies to curb erroneous host defence activity using peptides.

As integral part of humoral innate immunity, the complement system serves as fast-reacting pathogen and danger sensor that recognizes a broad spectrum of non-self (e.g. bacteria) and altered-self surfaces (e.g. apoptotic cells) and induces an appropriate immune response. ${ }^{[15]}$ The detection of molecular patterns such as antibody complexes or microbial carbohydrate signatures triggers an activation cascade that involves some 50 proteins, including serine proteases, receptors and regulators, and generates potent effectors that lead to lytic or phagocytic cell removal and stimulate downstream immune responses (Fig. 3A). The spatio-temporarily controlled activation of three orthologous plasma proteins (i.e. $\mathrm{C} 3, \mathrm{C} 4, \mathrm{C} 5$ ) by protease complexes is central to this process. $\mathrm{C} 3$ convertases assembling on activating surfaces cleave $\mathrm{C} 3$ into the chemoattractant $\mathrm{C} 3 \mathrm{a}$ and the opsonin $\mathrm{C} 3 \mathrm{~b}$, which covalently binds to the surface through its thioester and tags the cell for immune processing. C3b can form new C3 convertases, which fuels an amplification loop and generates $\mathrm{C} 5$ convertases that activate C5 to produce the anaphylatoxin C5a and initiate the generation of lytic membrane-attack complexes (MAC). While host cells are typically protected from complement attack by regulators, any disruption of the delicate activation-regulation balance may cause clinical complications. ${ }^{[16]}$ For example, a lack of regulators on erythrocytes leads to the rare yet severe haemolytic disease paroxysmal nocturnal haemoglobinuria (PNH). During organ transplantation, hypoxia leads to complement-mediated ischemia-reperfusion injury by exposing damage-associated patterns, whereas binding of antibodies to the foreign organ triggers a complement response that may lead to organ rejection. ${ }^{[16]}$

Despite the strong disease involvement of complement, the therapeutic arsenal is currently restricted to antibodies (i.e. eculizumab, ravulizumab) that act as PPI inhibitors of C5 activation, thereby preventing the generation of C5a and MAC. ${ }^{[17]}$ While successfully used in PNH and other diseases, these antibodies are expensive and ill-suited for self-administration. Moreover, C5 may not be the ideal target for certain indications. Owing to the prevalence of PPI within the complement cascade, therapeutic peptides have emerged as intriguing options for complement inhibition. Indeed, macrocyclic peptides preventing the activation of $\mathrm{C} 3$ (i.e. pegcetacoplan, AMY-101), C5 (i.e. zilucoplan) or C5a receptor 1 (i.e. ALS205), or impairing initiation steps (i.e. RLS-0071) are in active clinical evaluation (Fig. 3A). [17a] Several administration options, including subcutaneous, intravitreal and even oral delivery are considered for complement-targeted peptides.
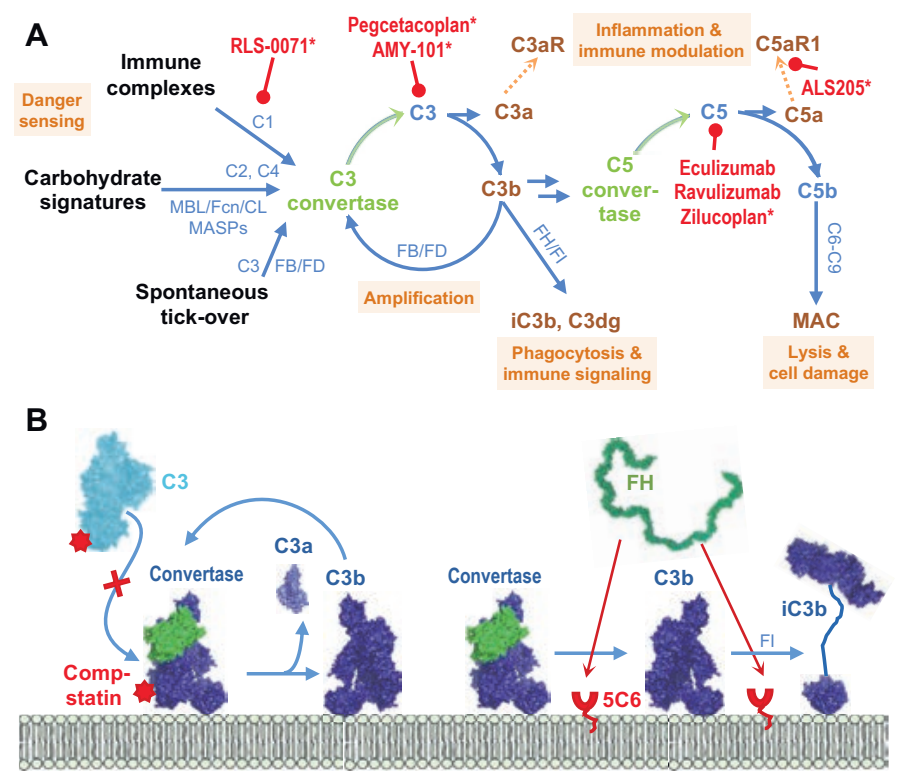

Fig. 3. A) Schematic overview of the complement system. Recognition of pathogen- or damage-associated signatures induces a cascade that generates inflammatory mediators and helps eliminating intruders. Yet, complement activation on host cells or biomedical surfaces contributes to various clinical conditions, thereby providing an attractive target for therapeutic interaction. Alongside two approved drugs (i.e. eculizumab, ravulizumab), several complement-targeted peptide therapeutics are currently in clinical evaluation (marked with an asterisk). B) In addition to blocking complement activation in circulation (e.g. by compstatin), biomedical surfaces may be protected using peptide coatings (e.g. 5C6) that attract host regulators such as factor $\mathrm{H}(\mathrm{FH})$.

Alongside their peptidic nature, these drug candidates all have in common that they block complement in circulation. ${ }^{[17 a]}$ At least in biomaterial- or transplant-associated reactions, preventing complement activation directly on the foreign surface may be more efficient. Moreover, this approach has the advantage to reduce complement activation in situ without concomitant systemic complement inhibition. Our group therefore develops peptidebased surface coatings to recruit circulating host regulators to biomedical surfaces and protect them from complement attack (Fig. 3B). Notably, a similar strategy is employed by various pathogens that express regulator-binding proteins as part of their immune evasion mechanism; as major complement regulator in solution and potent inhibitor of the central amplification loop, factor $\mathrm{H}$ $(\mathrm{FH})$ is a particularly attractive target. ${ }^{[18]}$ Following this natureinspired approach, a 14-amino-acid long, cyclic peptide (termed 5C6; ASSSR[CTYSHWC]SH) was discovered by phage display screening. 5C6 showed nanomolar affinity for FH and was able to recruit FH to biomedical surfaces without affecting the regulator's functional activity. ${ }^{[19]}$ The potential of 5C6 could be demonstrated in models where phospholipid-coupled 5C6 was inserted into cell membranes and impaired complement activation on erythrocytes and endothelial cells. ${ }^{[19,20]}$ Currently, we are performing in-depth SAR studies to evaluate the importance of key residues, cyclization options and tethering strategies for the efficacy and stability of the peptide coating, with the aim to assess 5C6-derived options in preclinical models of complement-related diseases.

\section{From Discovery to Clinic: The Compstatin Experience}

The immense potential of peptide-focused academic drug discovery can be exemplified by the development of the compstatin family of C3 inhibitors (Fig. 4). The initial lead compound, compstatin, was identified at the University of Pennsylvania in 1996 using phage display technology.[21] While screening a li- 
Compstatin $(\mathrm{KD} \sim 10 \mu \mathrm{M})$

I [ CVVQDWGHHRC] T

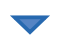

$\mathrm{Cp} 01(\mathrm{KD}=150 \mathrm{nM})$

AC-I [ CVWQDWGAHRC ] T

$\nabla$

$\mathrm{Cp05}(\mathrm{KD}=12 \mathrm{nM})$

AC-I [ CV ( 1MeW) QDWGAHRC] T

$\nabla$

$\mathrm{Cp} 40(\mathrm{KD}=0.5 \mathrm{nM})$

YI [ CV ( 1MeW) QDWSarAHRC] mI
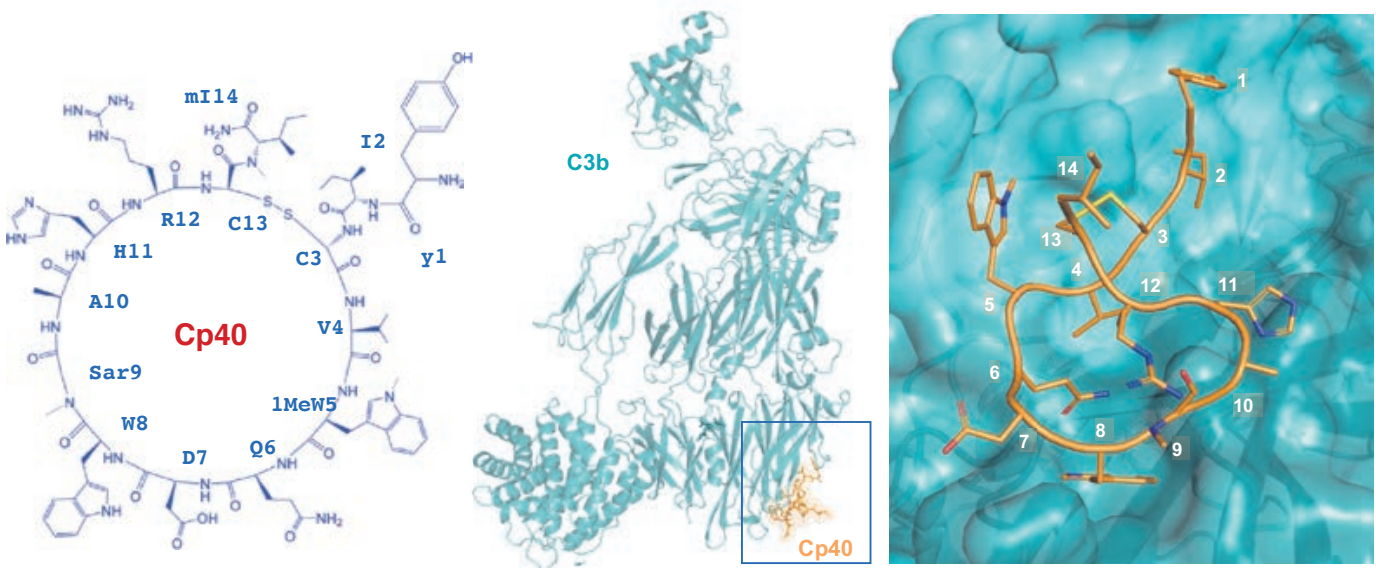

Fig. 4. Development of the compstatin family of peptidic C3 inhibitors. Starting from the initial lead, sequence optimization led to derivatives with largely improved affinity, resulting in the subnanomolar analog $\mathrm{Cp} 40$. Compstatin analogs tightly fit into a shallow binding site in the macroglobulin core of $\mathrm{C} 3$ and $\mathrm{C} 3 \mathrm{~b}$, which mediates the interaction of $\mathrm{C} 3$ with the convertase.

brary of 27 mer peptides against $\mathrm{C} 3 \mathrm{~b}$, a 13 amino acid sequence containing one disulphide bridge was identified as potent binding motif. Initially guided by alanine scans and NMR structures, stepwise optimization through $\mathrm{N}$-terminal acetylation and mutation of positions 4 and 9 resulted in the first nanomolar lead analogue Cp01.[22] Solving the crystal structure of target-bound Cp01 facilitated lead development and suggested that compstatin analogues act as PPI inhibitors that prevent the binding of C3 to the convertase, thereby impairing opsonization, amplification and effector generation. ${ }^{[23]}$ The introduction of a non-proteinogenic amino acid (1-methyl-Trp) at position 4 produced analogue $\mathrm{Cp} 05$ with $12 \mathrm{nM}$ affinity and good plasma stability, ${ }^{[24]}$ while backbone $\mathrm{N}$-methylation scans and $\mathrm{N}$-terminal extension revealed next-generation compstatin derivatives ( $\mathrm{Cp} 10-\mathrm{Cp} 40)$ with enhanced target residence and PK profiles. ${ }^{[25]}$ Thanks to strong academic collaboration, and despite a narrow species specificity for human/primate complement, compstatin derivatives were successfully evaluated in various preclinical models, which fuelled clinical development efforts.

Pegcetacoplan (Apellis), containing two Cp05 molecules bridged by a $40-\mathrm{kDa}$ PEG moiety, has recently shown superiority to eculizumab in phase 3 clinical trials in PNH and is also developed for age-related macular degeneration and other indications. ${ }^{[26]}$ Owing to its subnanomolar target affinity and beneficial PK properties, the Cp40-based candidate drug AMY-101 (Amyndas) can be administered in a non-PEGylated form and is currently being evaluated in phase 2 clinical trials for gingivitis and in COVID-19 patients.[27] Alongside its clinical success, compstatin provides an ideal model to explore optimization strategies for peptide therapeutics, including the introduction of albumin-binding tags, cystathionine bridges or charge/solubility modulators, among other examples. ${ }^{[28]}$ Finally, recent SAR studies by our group on the interaction between Cp40 and C3b (Fig. 4) revealed surprising roles for structural water and intramolecular peptide interactions that may guide the future development of this promising drug class (Lamers et al., under review).

\section{Conclusion and Outlook}

Peptide therapeutics have not only evolved into a rapidly emerging drug class with numerous clinical applications, particularly as PPI inhibitors to modulate erroneous host defence reactions, but also present an educational and experimental treasure chest for academic drug discovery and development. Recent advances in phage display, peptidomimetic design, targeting and peptide drug delivery paint a bright picture of the journey ahead.

\section{Acknowledgements}

The practical course 'Modern Drug Design' was introduced to the B.Sc. Pharmazeutische Wissenschaften of the University of Basel by Prof. em. Beat Ernst and Prof. Angelo Vedani†. In addition to the authors, Prof. Markus Lill, PD Dr. Martin Smieško, PD Dr. Said Rabbani, and several assistants of the Molecular Pharmacy and Computational Pharmacy groups at the Department of Pharmaceutical Sciences are involved in the current curricula. The compstatin and 5C6 technologies were discovered in the laboratory of Prof. John D. Lambris (University of Pennsylvania, Philadelphia, USA) and licenced to Apellis and Amyndas Pharmaceuticals. This work was supported by a grant from the Swiss National Science Foundation (31003A_176104 to DR).

Received: April 15, 2021

[1] J. L. Lau, M. K. Dunn, Bioorg. Med. Chem. 2018, 26, 2700, https://doi.org/10.1016/j.bmc.2017.06.052.

[2] M. Muttenthaler, G. F. King, D. J. Adams, P. F. Alewood, Nat. Rev. Drug Discov. 2021, 20, 309, https://doi.org/10.1038/s41573-020-00135-8.

[3] C. Sohrabi, A. Foster, A. Tavassoli, Nat. Rev. Chem. 2020, 4, 90, https://doi.org/10.1038/s41570-019-0159-2.

[4] G. P. Smith, Science 1985, 228, https://doi.org/10.1126/science.4001944.

[5] A. E. Nixon, D. J. Sexton, R. C. Ladner, MAbs 2014, 6, 73, https://doi.org/10.4161/mabs.27240.

[6] C. Heinis, T. Rutherford, S. Freund, G. Winter, Nat. Chem. Biol. 2009, 5 , 502, https://doi.org/0.1038/nchembio.184.

[7] Y. Huang, M. M. Wiedmann, H. Suga, Chem. Rev. 2019, 119, 10360, https://doi.org/10.1021/acs.chemrev.8b00430.

[8] A. Vedani, O. Schwardt, S. Rabbani, B. Ernst, Chimia 2006, 60, 70, https://doi.org/10.2533/000942906777675137

[9] a) K. E. Bernstein, Z. Khan, J. F. Giani, D. Y. Cao, E. A Bernstein, X. Z. Shen, Nat. Rev. Nephrol. 2018, 14, 325, https://doi.org/10.1038/nrneph.2018.15; b) K. E. Bernstein, F. S. Ong, W. L. Blackwell, K. H. Shah, J. F. Giani, R. A. Gonzalez-Villalobos, X. Z. Shen, S. Fuchs, R. M. Touyz, Pharmacol. Rev. 2013, 65, 1 , https://doi.org/10.1124/pr.112.006809.

[10] E. G. Erdös, Faseb J. 2006, 20, 1034, https://doi.org/10.1096/fj.06-0602ufm.

[11] D. W. Cushman, M. A. Ondetti, Nat. Med. 1999, 5, 1110 , https://doi.org/10.1038/13423.

[12] J. F. Waterfall, Br. J. Clin. Pharmacol. 1989, 27 Suppl 2, 139s, https://doi.org/10.1111/j.1365-2125.1989.tb03475.x.

[13] D. Ricklin, J. D. Lambris, Immunol. Rev. 2016, 274, 5, https://doi.org/10.1111/imr.12497.

[14] L. Lupu, A. Palmer, M. Huber-Lang, Front. Immunol. 2020, 11, 584514, https://doi.org/10.3389/fimmu.2020.584514.

[15] D. Ricklin, G. Hajishengallis, K. Yang, J. D. Lambris, Nat. Immunol. 2010 11, 785, https://doi.org/10.1038/ni.1923.

[16] D. Ricklin, E. S. Reis, J. D. Lambris, Nat. Rev. Nephrol. 2016, 12, 383, https://doi.org/10.1038/nrneph.2016.70.

[17] a) D. C. Mastellos, D. Ricklin, J. D. Lambris, Nat. Rev. Drug Discov. 2019 18, 707, https://doi.org/10.1038/s41573-019-0031-6; b) D. Ricklin, D. 
C. Mastellos, E. S. Reis, J. D. Lambris, Nat. Rev. Nephrol. 2018, 14, 26 , https://doi.org/10.1038/nrneph.2017.156.

[18] C. Q. Schmidt, J. D. Lambris, D. Ricklin, Immunol. Rev. 2016, 274, 152 , https://doi.org/10.1111/imr.12475.

[19] Y. Q. Wu, H. Qu, G. Sfyroera, A. Tzekou, B. K. Kay, B. Nilsson, K. Nilsson Ekdahl, D. Ricklin, J. D. Lambris, J. Immunol. 2011, 186, 4269, https://doi.org/10.4049/jimmunol.1003802.

[20] P. H. Nilsson, K. N. Ekdahl, P. U. Magnusson, H. Qu, H. Iwata, D. Ricklin, J. Hong, J. D. Lambris, B. Nilsson, Y. Teramura, Biomaterials 2013, 34, 985 , https://doi.org/10.1016/j.biomaterials.2012.10.040.

[21] A. Sahu, B. K. Kay, J. D. Lambris, J. Immunol. 1996, 157, 884.

[22] a) D. Morikis, N. Assa-Munt, A. Sahu, J. D. Lambris, Protein Sci. 1998 , 7, 619, https://doi.org/10.1002/pro.5560070311; b) A. Sahu, A. M. Soulika, D. Morikis, L. Spruce, W. T. Moore, J. D. Lambris, J. Immunol. 2000, 165, 2491, https://doi.org/10.4049/jimmunol.165.5.2491; c) B. Mallik, M. Katragadda, L. A. Spruce, C. Carafides, C. G. Tsokos, D. Morikis, J. D. Lambris, J. Med. Chem. 2005, 48, 274, https://doi.org/10.1021/jm0495531.

[23] B. J. Janssen, E. F. Halff, J. D. Lambris, P. Gros, J. Biol. Chem. 2007, 282, 29241, https://doi.org/10.1074/jbc.M704587200.

[24] M. Katragadda, P. Magotti, G. Sfyroera, J. D. Lambris, J. Med. Chem. 2006, 49, 4616, https://doi.org/10.1021/jm0603419.

[25] a) H. Qu, P. Magotti, D. Ricklin, E. L. Wu, I. Kourtzelis, Y. Q. Wu, Y. N. Kaznessis, J. D. Lambris, Mol. Immunol. 2011, 48, 481, D https://doi.org/10.1016/j.molimm.2010.10.004; b) H. Qu, D. Ricklin, H. Bai, H. Chen, E. S. Reis, M. Maciejewski, A. Tzekou, R. A. DeAngelis, R R. Resuello, F. Lupu, P. N. Barlow, J. D. Lambris, Immunobiology 2013, 218, 496, https://doi.org/10.1016/j.imbio.2012.06.003.

[26] P. Hillmen, J. Szer, I. Weitz, A. Röth, B. Höchsmann, J. Panse, K. Usuki, M. Griffin, J. J. Kiladjian, C. de Castro, H. Nishimori, L. Tan, M. Hamdani, P.
Deschatelets, C. Francois, F. Grossi, T. Ajayi, A. Risitano, R. P. de la Tour, N. Engl. J. Med. 2021, 384, 1028, https://doi.org/10.1056/NEJMoa2029073.

[27] D. C. Mastellos, B. G. P. Pires da Silva, B. A. L. Fonseca, N. P. Fonseca, M. Auxiliadora-Martins, S. Mastaglio, A. Ruggeri, M. Sironi, P. Radermacher, A. Chrysanthopoulou, P. Skendros, K. Ritis, I. Manfra, S. Iacobelli, M Huber-Lang, B. Nilsson, D. Yancopoulou, E. S. Connolly, C. Garlanda, F Ciceri, A. M. Risitano, R. T. Calado, J. D. Lambris, Clin. Immunol. 2020, 220, 108598, https://doi.org/10.1016/j.clim.2020.108598.

[28] a) N. Berger, T. D. Alayi, R. R. G. Resuello, J. V. Tuplano, E. S. Reis, J. D. Lambris, J. Med. Chem. 2018, 61, 6153, https://doi.org/10.1021/acs.jmedchem.8b00560; b) Y. Huang, E. S. Reis, P. J. Knerr, W. A. van der Donk, D. Ricklin, J. D. Lambris, ChemMedChem 2014, 9, 2223, https://doi.org/10.1002/cmdc.201402212; c) P. J. Knerr, A. Tzekou, D. Ricklin, H. Qu, H. Chen, W. A. van der Donk, J. D. Lambris, ACS Chem. Biol. 2011, 6, 753, https://doi.org/10.1021/cb2000378.

\section{License and Terms}

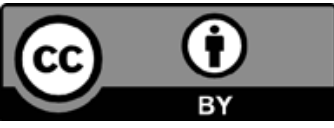

This is an Open Access article under the terms of the Creative Commons Attribution License CC BY 4.0. The material may not be used for commercial purposes.

The license is subject to the CHIMIA terms and conditions: (http:// chimia.ch/component/sppagebuilder/?view=page\&id=12).

The definitive version of this article is the electronic one that can be found at https://doi.org/10.2533/chimia.2021.495 